OPTICS

\section{Light amplification by seeded Kerr instability}

\author{
G. Vampa, ${ }^{1,2 *} \dagger$ T. J. Hammond, ${ }^{1} \dagger$ M. Nesrallah, ${ }^{1}$ A. Yu. Naumov, ${ }^{3}$ \\ P. B. Corkum, ${ }^{1,3}$ T. Brabec ${ }^{\text {1* }}$
}

Amplification of femtosecond laser pulses typically requires a lasing medium or a nonlinear crystal. In either case, the chemical properties of the lasing medium or the momentum conservation in the nonlinear crystal constrain the frequency and the bandwidth of the amplified pulses. We demonstrate high gain amplification (greater than 1000$)$ of widely tunable $(0.5$ to 2.2 micrometers) and short (less than 60 femtosecond) laser pulses, up to intensities of 1 terawatt per square centimeter, by seeding the modulation instability in an $\mathrm{Y}_{3} \mathrm{Al}_{5} \mathrm{O}_{12}$ crystal pumped by femtosecond near-infrared pulses. Our method avoids constraints related to doping and phase matching and therefore can occur in a wider pool of glasses and crystals even at far-infrared frequencies and for single-cycle pulses. Such amplified pulses are ideal to study strong-field processes in solids and highly excited states in gases.

A mplification of mode-locked laser pulses typically proceeds through linear optical pumping of a gain medium followed by stimulated emission at lower photon energies than that of the pump. The medium stores the energy difference, and material damage limits the maximum amplified power. Recently, the quest for ever higher-average powers and broader wavelength tunability has resulted in the exploitation of optical parametric amplification $(1,2)-$ a second-order nonlinearity in which pump photons are split into pairs of conjugate lower-energy photons, without energy stored in the nonlinear crystal. However, phase matching and absorption of the pump and seed waves limits the center wavelength and the bandwidth of the amplified waves $(3,4)$. Improving phase matching and transparency requires intense development of new nonlinear crystals. We show that Kerr instability (5-7), a thirdorder optical nonlinearity resulting in an intensitydependent refractive index and common to any material, can compete with the more exploited parametric amplification.

The nonlinear Schrödinger equation with a Kerr third-order nonlinearity admits unstable solutions $(6,7)$; pumping a nonlinear medium with a sufficiently intense driver leads to weak waves that experience exponential gain as they propagate. When these weak waves are seeded from noise, the continuous wave breaks up into an unstable train of pulses $(6,8)$, leading to the disintegration of the spatial mode of an optical beam (9) or the formation of oceanic (10) and optical rogue waves (11). Despite the wide oc-

${ }^{1}$ Department of Physics, University of Ottawa, Ottawa, ON K1N 6N5, Canada. 'Stanford PULSE Institute, SLAC National Accelerator Laboratory, Menlo Park, CA 94025, USA

${ }^{3}$ National Research Council of Canada, Ottawa, ON K1A OR6, Canada.

*Corresponding author. Email: gvampa@stanford.edu (G.V.); thomas.brabec@uottawa.ca (T.B.) †These authors contributed equally to this work. currence of Kerr instabilities in physics, the theoretical understanding is still limited to instabilities at frequencies comparable with that of the pump (6). Recently, a fully analytical theory of Kerr instabilities valid for perturbations of arbitrary frequencies has been developed (7) that predicts appreciable gain over a broad spectral range between about zero and twice the pump frequency. Here, we experimentally demonstrate seeded amplification of femtosecond laser pulses in the visible and infrared spectral regions.

By seeding gain at different wavelengths, it is possible to amplify femtosecond laser pulses over more than an octave bandwidth (Fig. 1). A comparison is shown for seed spectra at three different central wavelengths $(0.6,1.4$, and $1.8 \mu \mathrm{m})$ (Fig. 1, pale colored solid lines) with the corresponding amplified spectra (Fig. 1, bright colors, solid lines). The gain medium is a $\mathrm{Y}_{3} \mathrm{Al}_{5} \mathrm{O}_{12}$ (YAG) crystal pumped by long femtosecond pulses from a Ti:sapphire laser. Gain in excess of 1000 is achieved throughout the whole bandwidth spanning 0.5 to $2.2 \mu \mathrm{m}$ by simply adjusting the angle $\theta$ (Fig. 1) between the seed and the pump beams. Not only can the gain be tuned to the desired wavelength, but the spectral bandwidth is also rather large. At $1.4 \mu \mathrm{m}$, for example, the $100-\mathrm{nm}$ bandwidth of the seed is fully amplified. Despite the strong nonlinearity and the complex propagation of the pump through the crystal, the seed pulses propagate and amplify without appreciable phase modulation. We measured the amplified pulses with second-harmonic frequency resolved optical gating (SH-FROG). As demonstrated in Fig. 1, insets A to D, the duration of the amplified pulses remains close to the transform limit of the seed, in any case $<60 \mathrm{fs}$, and can even be shorter than the seed by pumping with short pulses (fig. S3). Furthermore, the beam quality is preserved during amplification (fig. S1). Our newly developed theory suggests that single-cycle pulses can potentially be amplified by controlling the pump and seed wavelengths. Therefore, together with automatic phase matching demonstrated below, Kerr amplification offers an interesting new approach to amplifying single-cycle pulses in the visible $(12,13)$, infrared (14-16), and even multicycle pulses in the terahertz region (17), where amplification schemes are presently nonexistent.

To characterize the gain, in the mathematical limit of continuous-wave seed and pump beams and infinitely extended in space, the amplification

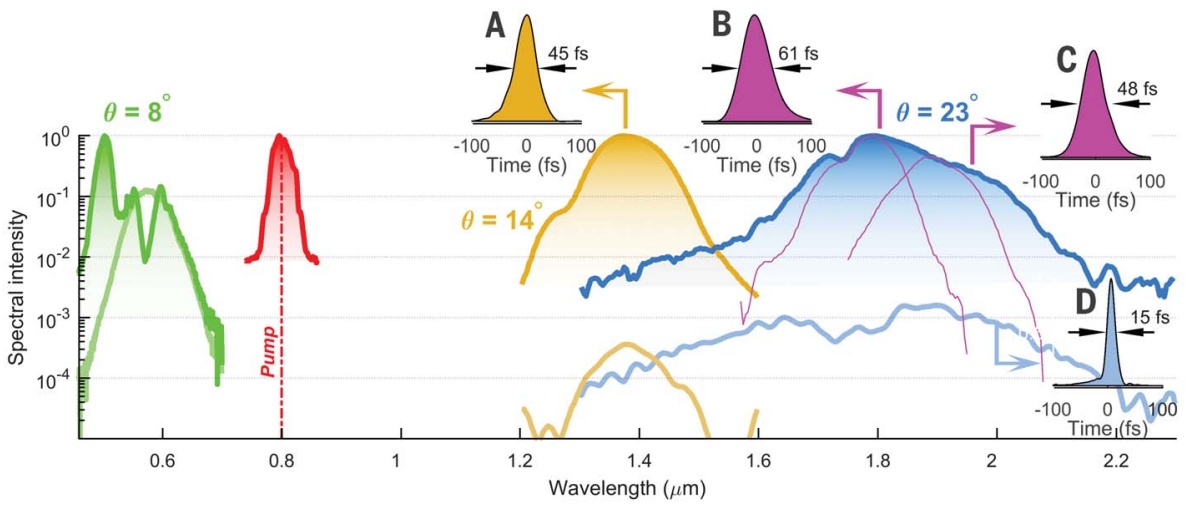

Fig. 1. Amplified spectra and pulse durations. Experimental amplified spectra (bright colors) and seed spectra (pale colors) for amplification around $0.5 \mu \mathrm{m}$ (green), $1.4 \mu \mathrm{m}$ (yellow), and $1.8 \mu \mathrm{m}$ (blue), normalized to corresponding amplified spectra. The pump spectrum at $0.8 \mu \mathrm{m}$ is shown in red. The angle between the pump and the seed laser beams $(\theta)$ is reported close to each amplified spectra. The pulse durations measured with a SHG-FROG are plotted in the insets for amplification spectra centered at (A) $1.4 \mu \mathrm{m},(\mathbf{B}) 1.75 \mu \mathrm{m}$, and (C) $1.95 \mu \mathrm{m}$ and (D) for the seed at $1.8 \mu \mathrm{m}$. The amplified pulses at $1.4 \mu \mathrm{m}$ preserve the seed pulse duration of $45 \mathrm{fs}$ [inset (A)]. Although the $1.8-\mu \mathrm{m}$ seed pulses last only $15 \mathrm{fs}$ (close to transform limit), the angular chirp of the amplification process narrows the spectrum that enters the FROG apparatus, which is positioned a few meters away from the YAG crystal. The measured spectra associated with insets (B) and (C) are plotted with purple lines. Each slice of the spectrum generates transform limited pulses (at $1.75 \mu \mathrm{m}$ the measured bandwidth is $76 \mathrm{~nm}$, and at $1.85 \mu \mathrm{m}$ it is $100 \mathrm{~nm}$ ). 
A

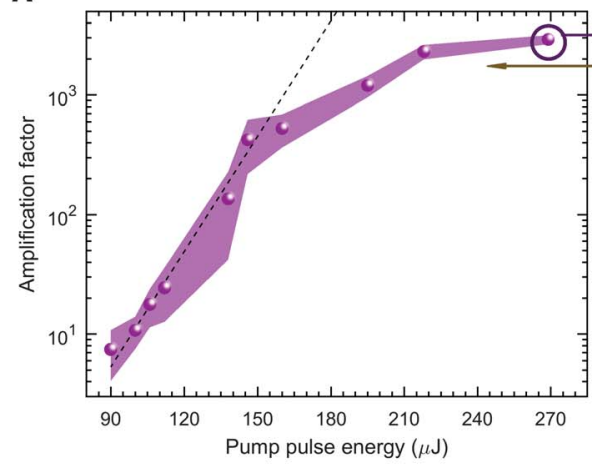

B

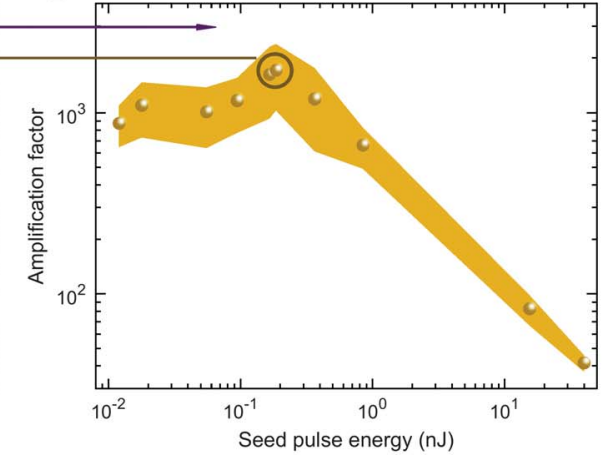

Fig. 2. Dependence of gain on pump and seed powers. (A) The amplification factor (the ratio between amplified and seed powers, spectrally integrated) increases exponentially with pump pulse energy, in agreement with theoretical expectation (black dotted line, which is reported in the supplementary materials) (18), but saturates for pump energies higher than $150 \mu \mathrm{J}$, which are close to the damage threshold of YAG $\left(\sim 300 \mu \mathrm{J}\right.$, corresponding to a vacuum intensity of $\left.10 \mathrm{TW} / \mathrm{cm}^{2}\right)$. (B) As a function of seed pulse energy, amplification remains high $(>1000)$ for seed energies $<0.2 \mathrm{~nJ}$ but decreases with a polynomial behavior for higher seed energies. Seed energy is varied at the maximum pump power (purple circles), whereas pump energy is varied at the seed energy just before the onset of saturation (yellow circle). The seed center wavelength is $1.4 \mu \mathrm{m}$.

Fig. 3. Angular chirp of the amplified spectrum. Amplified spectrum centered around $1.8 \mu \mathrm{m}$ as a function of propagation angle, measured in the far field. The angular chirp of $3 \mu \mathrm{m} / \mathrm{rad}$ and the emission angle agree with the theoretical prediction (gray dashed line). It is the angularly integrated spectrum that is plotted as a blue line in Fig. 1.

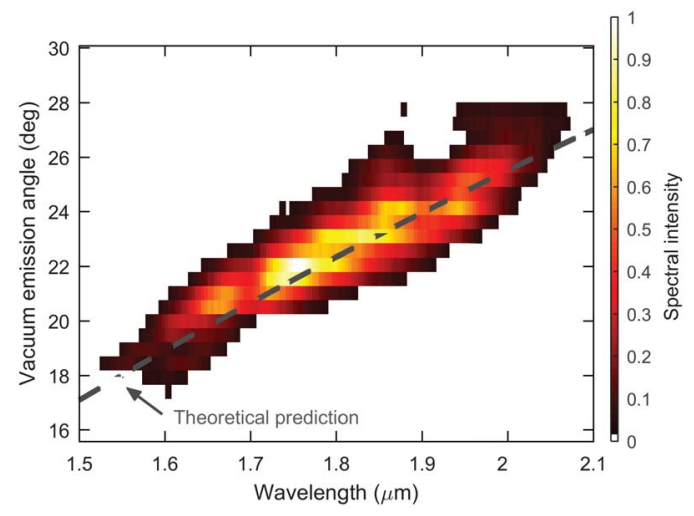

proceeds exponentially with the length of the crystal with intensity gain coefficient (7)

$$
\bar{g}(\Omega)=\frac{k_{n(+)} k_{n(-)}}{\sqrt{k_{\mathrm{p}}^{2}-\left(\sigma D_{u}\right)^{2}}}
$$

Here, $\Omega=\omega-\omega_{\mathrm{p}}$ is the frequency shift between seed $(\omega)$ and $\operatorname{pump}\left(\omega_{\mathrm{p}}\right), k_{n( \pm)}(\Omega)=\left(\omega_{\mathrm{p}} \pm \Omega\right) \sqrt{n_{2} I_{\mathrm{p}}} / c$ is the nonlinear wavevector, and $I_{\mathrm{p}}$ and $n_{2}$ are the pump intensity and the nonlinear refractive index, respectively. $k_{\mathrm{p}}^{2}=k^{2}\left(\omega_{\mathrm{p}}\right)+k_{n}^{2}(0)$ is the total wave vector of the pump given by adding the linear wave vector $k=\omega n(\omega) / c$ to the nonlinear wave vector at the pump wavelength. $\sigma=$ $\left[k_{v}\left(\omega_{\mathrm{p}}\right)+D_{g}\right] / k_{\mathrm{p}}$, where $k_{v}^{2}(\omega)=k^{2}(\omega)+2 k_{n}^{2}(\omega)$ is the seed wave vector (the nonlinearity is added twice compared with $k_{\mathrm{p}}$ ). $D_{\{g, u\}}$ takes into account the dispersion of the medium to any frequency shift (7). Amplification of finite pulses and Gaussian beams modifies this ideal gain factor by taking into account the temporal and spatial geometry (supplementary materials) (18).

We measured exponential growth with respect to pump intensity (Fig. 2A), in agreement with the theoretical prediction (Fig. 2A, dashed cesses, however, here phase matching is automatically satisfied (7). The measured angular chirp (Fig. 3) matches the theoretical prediction well. Because the chirp is almost linear with wavelength, it will be possible to compensate for the beam divergence that phase matching imposes with a dispersive element placed just after the medium. Furthermore, the angular chirp is only weakly dependent on the pump intensity (fig. S6), suggesting that it is uniform across the amplified beam profile. Matching the transverse momentum of optimum gain is a critical parameter to achieve amplification. Optimum amplification of a desired wavelength is achieved in the experiment by tuning the angle $\theta$ (Fig. 1). And as confirmed in Fig. 3, the measured optimum angle coincides with that predicted by theory.

By using near-infrared pump pulses, we have demonstrated seeded amplification by means of Kerr instability of short femtosecond pulses over a widely tunable spectral range. Amplification occurred in a crystal (YAG) whose nonlinear refractive index is of average magnitude. The nonlinearity is at least 100 times stronger in special materials such as chalcogenide glasses (21) or graphene (22) or at frequencies at which the linear permittivity approaches zero (23). In the latter case, a total index change of $170 \%$ has been observed. Therefore, high gain amplification seems feasible in only a few micrometers, if not nanometers, of these materials, with gain coefficients as high as $\bar{g} \sim 100 \mathrm{~s} \mathrm{~mm}^{-1}$ (as opposed to $\sim 5 \mathrm{~mm}^{-1}$, as in YAG).

Our theory predicts that gain terminates at frequencies for which either $\kappa_{\perp}^{2}<0$ or $k_{\mathrm{p}}^{2} \simeq\left(\sigma D_{u}\right)^{2}$. In $\mathrm{KBr}$ pumped by $2.1-\mu \mathrm{m}$ femtosecond pulses, the cut-off extends to $14 \mu \mathrm{m}(\sim 21 \mathrm{THz})$, and to $42 \mu \mathrm{m}(\sim 7.1 \mathrm{THz})$ in the CW limit, therefore up to far-infrared frequencies, at which nonlinear crystals perform poorly or amplification schemes are inexistent altogether. Neither linear gain media nor $\chi^{(2)}$ nonlinear crystals offer as wide a bandwidth tunability because of limitations in achieving phase matching (4) or because of narrow gain profiles. Several nonlinear crystals also absorb mid-infrared light (24).

The energy output can be scaled up to arbitrarily high energies by adapting chirped pulse amplification schemes $(19,20)$. Furthermore, the amplified bandwidth can sustain single-cycle pulses. Therefore, Kerr amplification will result in a shift of design paradigm for next-generation high-intensity laser sources. For the moment, the demonstrated microjoule-level pulse energies are already suitable to trigger strong-field processes in condensed matter-such as high harmonic generation (25), ablation and damage (26), optically driven currents (27), and photoemission (28)and to study highly excited Rydberg states (29).

Given the broad occurrence of Kerr nonlinear instabilities in nature $(10,11)$, our demonstration with femtosecond laser pulses may prove useful to address the spatiotemporal evolution of instabilities in forms other than light over a broad range of frequencies, where the effect of the full linear and nonlinear (23) dispersion of the medium becomes important. 


\section{REFERENCES AND NOTES}

1. R. Baumgartner, R. Byer, IEEE J. Quantum Electron. 15 , 432-444 (1979).

2. R. W. Boyd, Nonlinear Optics (Academic Press, 2003).

3. C. Manzoni, G. Cerullo, J. Opt. 18, 103501 (2016).

4. B. E. Schmidt et al., Nat. Commun. 5, 3643 (2014).

5. K. Tai, A. Tomita, J. L. Jewell, A. Hasegawa, Appl. Phys. Lett. 49, 236-238 (1986)

6. L. W. Liou, X. D. Cao, C. J. McKinstrie, G. P. Agrawal, Phys. Rev. A 46, 4202-4208 (1992).

7. M. Nesrallah et al., arXiv:1711.04417 [physics.optics] (2017)

8. K. Tai, A. Hasegawa, A. Tomita, Phys. Rev. Lett. 56, 135-138 (1986)

9. D. Kip, M. Soljacic, M. Segev, E. Eugenieva, D. N. Christodoulides, Science 290, 495-498 (2000).

10. C. Kharif, E. Pelinovsky, Eur. J. Mech. B 22, 603-634 (2003)

11. J. M. Dudley, F. Dias, M. Erkintalo, G. Genty, Nat. Photonics 8 , 755-764 (2014).

12. E. Goulielmakis et al., Science 320, 1614-1617 (2008).

13. A. Wirth et al., Science 334, 195-200 (2011).

14. G. Krauss et al., Nat. Photonics 4, 33-36 (2010)
15. P. Krogen et al., Nat. Photonics 11, 222-226 (2017).

16. H. Liang et al., Nat. Commun. 8, 141 (2017).

17. M. Tonouchi, Nat. Photonics 1, 97-105 (2007).

18. Materials and methods are available as supplementary materials

19. D. Strickland, G. Mourou, Opt. Commun. 55, 447-449 (1985).

20. A. Dubietis, G. Jonušauskas, A. Piskarskas, Opt. Commun. 88 437-440 (1992)

21. F. Smektala, C. Quemard, V. Couderc, A. Barthélémy, J. Non-Cryst. Solids 274, 232-237 (2000).

22. H. Zhang et al., Opt. Lett. 37, 1856-1858 (2012).

23. M. Z. Alam, I. De Leon, R. W. Boyd, Science 352, 795-797 (2016).

24. D. N. Nikogosyan, Nonlinear Optical Crystals: A Complete Survey (Springer Science \& Business Media, 2006).

25. S. Ghimire et al., Nat. Phys. 7, 138-141 (2011).

26. R. R. Gattass, E. Mazur, Nat. Photonics 2, 219-225 (2008)

27. A. Schiffrin et al., Nature 493, 70-74 (2013).

28. M. Krüger, M. Schenk, P. Hommelhoff, Nature 475, 78-81 (2011).

29. U. Eichmann, A. Saenz, S. Eilzer, T. Nubbemeyer, W. Sandner, Phys. Rev. Lett. 110, 203002 (2013).

\section{ACKNOWLEDGMENTS}

We acknowledge valuable financial support from the Air Force Office of Scientific Research (AFOSR) under award FA9550-16-1 0109, the AFOSR Multidisciplinary University Research Initiative grant FA9550-15-1-0037, and from Canada's National Research Council and Natural Sciences and Engineering Research Council. The authors declare no competing financial interests. G.V. thanks B. Schmidt for insightful discussions. All data needed to evaluate the conclusions in the paper are present in the paper and/or the supplementary materials.

\section{SUPPLEMENTARY MATERIALS}

www.sciencemag.org/content/359/6376/673/suppl/DC1 Materials and Methods

Supplementary Text

Figs. S1 to $\mathrm{S} 6$

References (30-34)

19 September 2017; accepted 21 December 2017

10.1126/science.aaq0053 


\section{Science}

\section{Light amplification by seeded Kerr instability}

G. Vampa, T. J. Hammond, M. Nesrallah, A. Yu. Naumov, P. B. Corkum and T. Brabec

Science 359 (6376), 673-675.

DOI: $10.1126 /$ science.aaq0053

\section{Seeding a laser amplifier}

Amplification of femtosecond laser pulses requires a lasing medium or a nonlinear crystal. The chemical properties of the lasing medium or adherence to momentum conservation rules in the nonlinear crystal constrain the frequency and the bandwidth of the amplified pulses. Vampa et al. seeded modulation instability in a laser crystal pumped with femtosecond near-infrared pulses. This provided a method for the high gain amplification of broadband and short laser pulses up to intensities of 1 terawatt per square centimeter. The method avoids constraints related to doping and phase matching and can be expected to be applied to a wide pool of glasses and crystals. Science, this issue p. 673

ARTICLE TOOLS

SUPPLEMENTARY MATERIALS

REFERENCES

PERMISSIONS http://science.sciencemag.org/content/359/6376/673

http://science.sciencemag.org/content/suppl/2018/02/07/359.6376.673.DC1

This article cites 29 articles, 4 of which you can access for free http://science.sciencemag.org/content/359/6376/673\#BIBL

http://www.sciencemag.org/help/reprints-and-permissions

Use of this article is subject to the Terms of Service

Science (print ISSN 0036-8075; online ISSN 1095-9203) is published by the American Association for the Advancement of Science, 1200 New York Avenue NW, Washington, DC 20005. 2017 (C) The Authors, some rights reserved; exclusive licensee American Association for the Advancement of Science. No claim to original U.S. Government Works. The title Science is a registered trademark of AAAS. 


\section{Science МIAAAS}

\section{Supplementary Material for}

\section{Light amplification by seeded Kerr instability}

G. Vampa,* T. J. Hammond, M. Nesrallah, A. Yu. Naumov, P. B. Corkum, T. Brabec*

*Corresponding author. Email: gvampa@stanford.edu (G.V.); thomas.brabec@uottawa.ca (T.B.)

Published 9 February 2018, Science 359, 673 (2017)

DOI: $10.1126 /$ science.aaq0053

This PDF file includes:

Materials and Methods

Supplementary Text

Figs. S1 to S6

References 


\section{Materials and Methods}

Femtosecond laser pulses centered around $0.8 \mu \mathrm{m}$ (the "pump") are loosely focused $(\sim \mathrm{f} / 200)$ in a $2 \mathrm{~mm}$ thick YAG crystal to a peak intensity in vacuum between 3 and 9 $\mathrm{TW} / \mathrm{cm}^{2}$. The seed beam is more strongly focused $(\sim \mathrm{f} / 10-\mathrm{f} / 30)$ than the pump. At these intensities, the pump beam becomes unstable during propagation in the crystal and a visible speckle pattern is observed at the output as the combined result of self-phase modulation and spatial modulation instability.

Two separate experiments have been conducted.

In the first experiment, a Ti:Sapphire laser delivering $\sim 1 \mathrm{~mJ}, 30 \mathrm{fs}$ pulses at $1 \mathrm{kHz}$ repetition rate has been used to amplify $0.5 \mu \mathrm{m}$ and $1.4 \mu \mathrm{m}$ pulses. A broadband seed spanning from $0.5 \mu \mathrm{m}$ to $1.6 \mu \mathrm{m}$ is obtained by self-phase modulation in a $3 \mathrm{~mm}$ thick sapphire plate with a small fraction of the pump energy. Amplification of $0.5 \mu \mathrm{m}$ pulses proceeds directly from this super-continuum. The seed at $1.4 \mu \mathrm{m}$ is obtained by parametric amplification of the infrared portion of the super-continuum with a fraction of the pump in a $3 \mathrm{~mm}$ thick type-II BBO crystal. Clean pulses of $45 \mathrm{fs}$ duration are generated. Seeds at $0.5 \mu \mathrm{m}$ and $1.4 \mu \mathrm{m}$ are then focused (f/30) into a $2 \mathrm{~mm}$ YAG crystal, where they overlap in space and time with the $0.8 \mu \mathrm{m}$ pump, whose beam size and power are controlled to optimize gain. The pump pulses used for the amplification have been stretched to 140 fs to avoid inducing phase modulation on the seed.

In the second experiment, a Ti:Sapphire laser delivering $16 \mathrm{~mJ}, 50 \mathrm{fs}$ pulses at 100 $\mathrm{Hz}$ repetition rate has been used to amplify the broadband spectrum around $1.8 \mu \mathrm{m}$. The broadband seed at $1.8 \mu \mathrm{m}$ is obtained by spectral broadening in a $1 \mathrm{~m}$ long hollow-core fiber of $1.3 \mathrm{~mJ}$ pulses obtained from a commercial Optical Parametric Amplifier (Light Conversion, HE-TOPAS-C) pumped by $7 \mathrm{~mJ}$ of $0.8 \mu \mathrm{m}$ pulses. The broadband seed can be compressed down to 15 fs ( 2.5 optical cycles), as measured with a dispersion-free SHG FROG. The seed is then focused ( $\mathrm{f} / 10)$ into the same YAG crystal of the first experiment, together with a fresh pump (whose beam size and power are controlled like in the first experiment). Again, the pump pulses have been stretched to $140 \mathrm{fs}$.

We have also achieved amplification in Fused Silica but with lower gain, due to the lower $\mathrm{n}_{2}$ and damage thresholds compared to YAG. This data is not presented here.

\section{Supplementary Text}

\section{Calculation of gain}

The scaling of the amplification factor with the intensity of the pump (black dashed line in Fig. 2A) is calculated as:

$$
\frac{W_{s}(l)}{W_{s}(0)}=\frac{W_{x}}{\sqrt{\operatorname{Re}\left\{q_{x}\right\}}} e^{\int_{0}^{l} \overline{\bar{g}}(z) d z}
$$

where $W_{s}(z)$ is the seed power, $w_{x}$ is the seed beam width, $q_{x}=w_{x}^{2}+2\left(g_{2}+i \alpha\right) l, \bar{g}(z)$ is the gain coefficient calculated with Eq. 1 , and $\alpha(\Omega)=\sigma D_{u} /\left[k_{p}^{2}-\left(\sigma D_{u}\right)^{2}\right]$. Because of the non-collinear geometry between seed and pump, the seed experiences inhomogeneous gain along its propagation direction " $z$ ". The inhomogeneous gain is encoded by varying the pump intensity $\mathrm{I}_{\mathrm{p}}$ as a function of ' $z$ ': 


$$
I_{p}(z)=I_{p} \exp \left[\frac{-2 z^{2}}{w_{p, e f f}^{2}}\right],
$$

where $I_{p}$ is the peak intensity in the middle of the pump beam. The integral runs over the overlap region between seed and pump. $g_{2}$ represents the width of the gain profile in momentum space. Both $g_{2}$ and $\alpha$ are defined in Ref. (7).

The length of the interaction ' $l$ ' is estimated by considering the propagation angle between the seed and the pump and the size of both beams. The propagation angle in air is $14^{\circ}$. This is reduced to $8.1^{\circ}$ upon refraction of the pump at the entrance surface of YAG. The waist diameter of the seed $\left(1 / \mathrm{e}^{2}\right)$ is $w_{x}=54 \mu \mathrm{m}$, the pump waist diameter is $226 \mu \mathrm{m}$, so the two beams overlap for $l=1.6 \mathrm{~mm}$, and the effective width of the pump beam seen by the seed is $w_{p, e f f}^{2}=880 \mu \mathrm{m}$.

Equation S1 is equivalent to Eq. (21) of Ref. (7), in the limit where only the geometric properties of the amplification are retained. That is, we set $\tau=\tau_{g}(l), \Gamma_{s}^{\prime}=$ $0, \bar{k}_{\perp}=\bar{k}_{\perp s}$. Technically, we equate the input and output pulse durations (in agreement with the experimental results at $1.4 \mu \mathrm{m}$ ), neglect dispersion of the gain coefficient, and we set the angle between seed and pump to optimize amplification of the seed center wavelength (like in the experiment). Compared to Ref. (7), we introduce the integral over the distance in the exponent, but neglect it in the prefactor. We find that, although the momentum overlap between gain and seed beam changes with distance - and would be encoded in a z-dependent $q_{x}(z)$ - the prefactor contributes little to the general exponential trend. Much more important is taking proper account of the $\mathrm{z}$-dependent gain, which almost halves the gain coefficient (and therefore the slope of the black dotted line in Fig. 2A) compared to the CW case of Eq. 1.

Other parameters required to plot the theoretical scaling are pump wavelength $\lambda_{p}=$ $0.8 \mu \mathrm{m}$, seed wavelength $\lambda_{s}=1.4 \mu \mathrm{m}$, nonlinear index of YAG $n_{2}=6.2 \times 10^{-16} \mathrm{~cm}^{2} / \mathrm{W}$ (30). The linear refractive index of YAG is taken from Ref. (31). The Sellmeier expansion is:

$$
n^{2}-1=\frac{2.282 \lambda^{2}}{\lambda^{2}-0.01185}+\frac{3.27644 \lambda^{2}}{\lambda^{2}-282.734}
$$

The group velocity at the pump wavelength $(800 \mathrm{~nm})$ is $+97 \mathrm{fs}^{2} / \mathrm{mm}$. The intensities in Fig. 2 range from $3-9 \mathrm{TW} / \mathrm{cm}^{2}$.

Spatial profile of amplified beam

Despite the exponential dependence of the amplified pulse energy on the pump, and the strong spatial modulation on the pump, the amplified beam is rather clean. To demonstrate this, the beam at $1.4 \mu \mathrm{m}$ has been frequency doubled in a BBO crystal and the focus of the second harmonic imaged (Fig. S1). Without the BBO, or when either the seed or the pump is blocked, the beam disappears, indicating that it is the amplified 1.4 $\mu \mathrm{m}$ that is being frequency doubled.

$\underline{\text { Bandwidth }}$ of the amplified pulses 
The spectral bandwidth of the amplified pulses is largely determined by how well the transverse momentum of each wavelength in the seed spectrum overlaps with the gain bandwidth for that wavelength. The maximum of the latter shifts with wavelength and results in angular chirp of the amplified spectrum. Therefore, one way to increase the amplified spectral bandwidth is to propagate each wavelength at the optimum angle with respect to the pump beam. A similar approach has been employed in $\chi^{(2)}$ amplification, and is referred to as "achromatic phase matching" (32).

Alternatively, one could increase the width of the momentum distribution of the seed beam by focusing the beam more strongly. This is demonstrated in Fig. S2, where the amplified bandwidth around $0.5 \mu \mathrm{m}$ is increased from $20 \mathrm{~nm}$ with $\mathrm{f}=30 \mathrm{~cm}$ to $30 \mathrm{~nm}$ with $\mathrm{f}=15 \mathrm{~cm}$. The drawback is that the intensity in the seed beam also increases, which may result in earlier saturation of the amplification process and lower amplified pulse energy.

Broad amplified bandwidths can even be generated directly during amplification by pumping with short pump pulses and narrow-band seed pulses. The instantaneous nature of the $\chi^{(3)}$ response of YAG assures that amplification takes place only when the pump is present. To demonstrate this effect, a $10 \mathrm{~nm}$ bandwidth-limited seed around $0.5 \mu \mathrm{m}$ is amplified to $16 \mathrm{~nm}$ bandwidth pulses, consistent with the $30 \mathrm{fs}$ duration of the pump (see Fig. S3). Near-infrared pulses of $<5$ fs duration are routinely achieved in attosecond laboratories (12). They can be used to generate and amplify few-cycle pulses at midinfrared wavelengths from near-CW seed beams, thereby overcoming the challenging task (4) of directly amplifying octave-spanning bandwidths with second-order nonlinearities $(33,34)$. In the limiting case, truly $\mathrm{CW}$ lasers can potentially be pulsed as well.

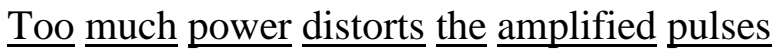

Figure S4 shows the spectrum of the amplified pulses for increasing seed pulse energy. When the seed energy is above $40 \mathrm{~nJ}$, the amplified spectrum is broadened significantly (red line in panel A), most likely from cross-phase modulation with the pump. The amplified energy of $1.6 \mu \mathrm{J}$ corresponds to an intensity of $3.5 \mathrm{TW} / \mathrm{cm}^{2}$. Moreover, it seems that the spectral phase acquires a higher third-order contribution (dashed lines in panel B), which renders the pulse more asymmetric (panel $\mathbf{C}$ ). Cross-talk between the two similarly intense beams is observed in the suppression of the visible speckle pattern generated by the pump beam. Due to the exponential sensitivity of the amplification process on the pump intensity, we speculate that depletion of the pump beam along the interaction length quenches amplification.

\section{Dependence of angle of optimum gain on pump wavelength}

As discussed in the text, optimum amplification occurs only if the lateral momentum of the seed coincides with the lateral momentum of maximum gain, $\bar{k}_{\perp}$. The angle that the maximally amplified seed forms with the pump, which coincides with the emission angle in our experiment (dashed gray line in Fig. 3), can be calculated with $\sin \theta=$ 
$\kappa_{\perp} /\left(k_{p}+\sigma D_{u}\right)$. The variation of this angle with wavelength of the seed represents the angular chirp of the amplified beam. Like $\bar{k}_{\perp}$, the chirp depends on the frequency shift $\Omega$ between the seed and pump wavelengths. Figure S5 shows how the chirp in the infrared to mid-infrared spectral regions is halved by pumping at $1.03 \mu \mathrm{m}$, rather than at $0.8 \mu \mathrm{m}$.

Furthermore, Fig. S6 show that the theoretical angular chirp varies only weakly with the pump intensity. Here, the difference in the higher and lower intensities corresponds to a drop of the amplification factor by $99 \%$. Therefore, our calculation suggests that the angular chirp is uniform across the amplified beam profile - a necessary condition for its compensation with simple optical elements. 


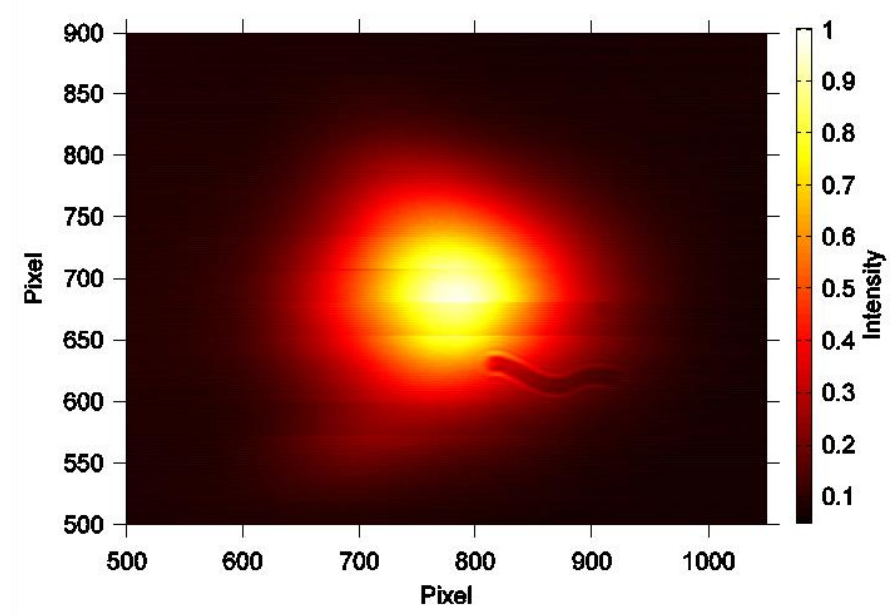

Fig. S1.

Amplified beam quality. Image of the second harmonic of the amplified beam at 1.4 $\mu \mathrm{m}$, taken at the focus. The image indicates that the beam quality of the seed is preserved during amplification. 


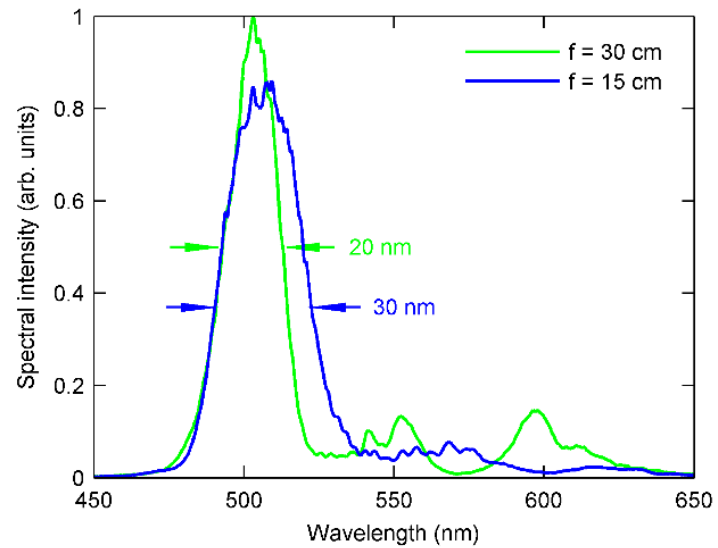

Fig. S2.

Seed focusing controls the amplified bandwidth. The amplified bandwidth increases from $20 \mathrm{~nm}$ to $30 \mathrm{~nm}$ when the seed focus length decreases from $30 \mathrm{~cm}$ to $15 \mathrm{~cm}$. 


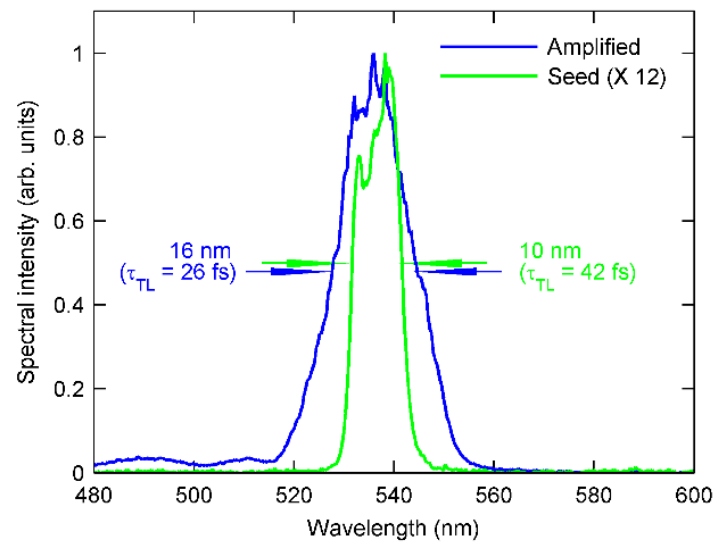

Fig. S3.

Short pump pulses increase the amplified bandwidth. When the pump pulses (of $30 \mathrm{fs}$ duration) are shorter than the seed pulses (green line, transform limit of $42 \mathrm{fs}$ ), the amplified spectrum (blue line) is broadened to sustain pulses as short as the pump (transform limit of $26 \mathrm{fs}$ ). 

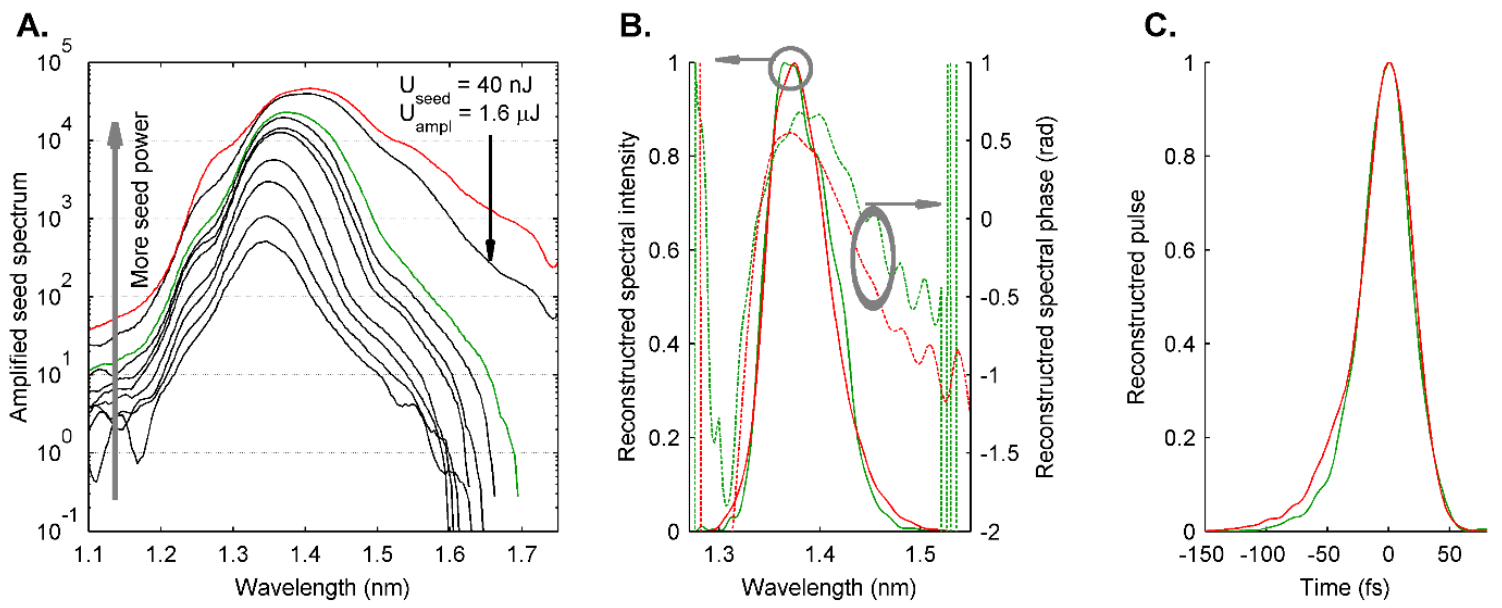

Fig. S4.

High seed energy broadens the amplified spectrum. The amplified spectrum is excessively broadened for seed pulse energies above $40 \mathrm{~nJ}$ (red trace in panel A). The broadening is associated with increased third-order spectral phase (red dashed line in panel B) with respect to lower seeding pulse energy (dashed green line in panel B). In the temporal domain (panel $\mathbf{C}$ ), the amplified pulse becomes slightly asymmetric (red line compared to green line). The spectral amplitude and phase and the pulse duration have been reconstructed with a dispersion-free SHG FROG. 


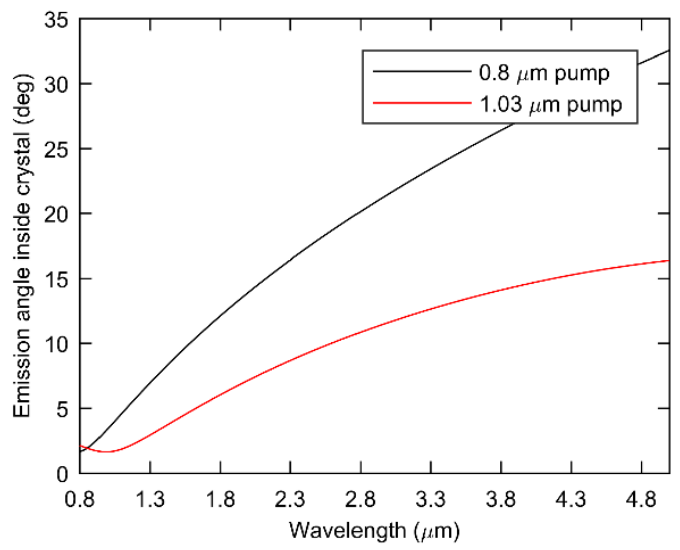

\section{Fig. S5.}

Dependence of emission angle with pump wavelength. The emission angle (inside the crystal) in the infrared and mid-infrared spectral region can be halved by pumping at 1.03 $\mu \mathrm{m}$ (red line) rather than at $0.8 \mu \mathrm{m}$ (black line). 


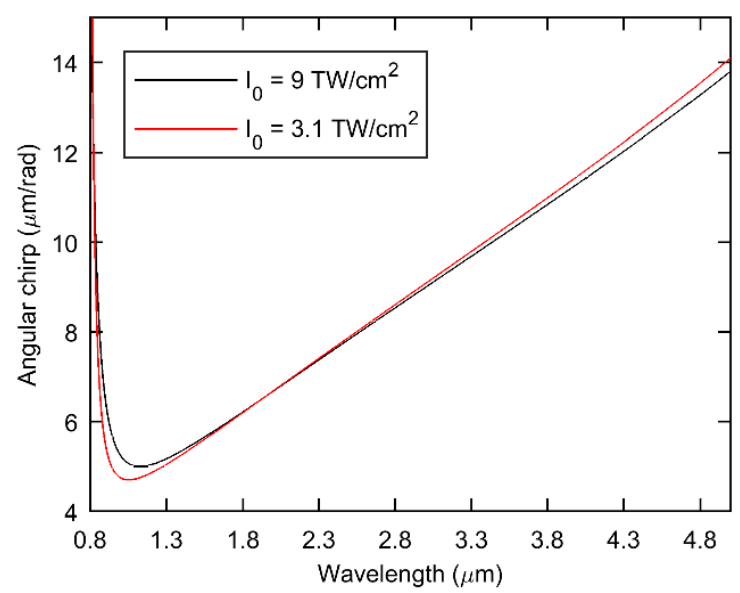

Fig. S6.

Dependence of the angular chirp on pump intensity. The angular chirp varies weakly by decreasing the pump intensity. The calculated chirp is for pump center wavelength at $0.8 \mu \mathrm{m}$, and for a YAG crystal (the parameters are given in the supplementary text). The highest pump intensity $\left(9 \mathrm{TW} / \mathrm{cm}^{2}\right)$ corresponds to a pump energy of $270 \mu \mathrm{J}$. 


\section{References and Notes}

1. R. Baumgartner, R. Byer, Optical parametric amplification. IEEE J. Quantum Electron. 15, 432-444 (1979). doi:10.1109/JQE.1979.1070043

2. R. W. Boyd, Nonlinear Optics (Academic Press, 2003).

3. C. Manzoni, G. Cerullo, Design criteria for ultrafast optical parametric amplifiers. J. Opt. 18, 103501 (2016). doi:10.1088/2040-8978/18/10/103501

4. B. E. Schmidt, N. Thiré, M. Boivin, A. Laramée, F. Poitras, G. Lebrun, T. Ozaki, H. Ibrahim, F. Légaré, Frequency domain optical parametric amplification. Nat. Commun. 5, 3643 (2014). Medline

5. K. Tai, A. Tomita, J. L. Jewell, A. Hasegawa, Generation of subpicosecond solitonlike optical pulses at $0.3 \mathrm{THz}$ repetition rate by induced modulational instability. Appl. Phys. Lett. 49, 236-238 (1986). doi:10.1063/1.97181

6. L. W. Liou, X. D. Cao, C. J. McKinstrie, G. P. Agrawal, Spatiotemporal instabilities in dispersive nonlinear media. Phys. Rev. A 46, 4202-4208 (1992). doi:10.1103/PhysRevA.46.4202 Medline

7. M. Nesrallah et al., arXiv:1711.04417 [physics.optics] (2017).

8. K. Tai, A. Hasegawa, A. Tomita, Observation of modulational instability in optical fibers. Phys. Rev. Lett. 56, 135-138 (1986). doi:10.1103/PhysRevLett.56.135 Medline

9. D. Kip, M. Soljacic, M. Segev, E. Eugenieva, D. N. Christodoulides, Modulation instability and pattern formation in spatially incoherent light beams. Science 290, 495-498 (2000). doi:10.1126/science.290.5491.495 Medline

10. C. Kharif, E. Pelinovsky, Physical mechanisms of the rogue wave phenomenon. Eur. J. Mech. B 22, 603-634 (2003). doi:10.1016/j.euromechflu.2003.09.002

11. J. M. Dudley, F. Dias, M. Erkintalo, G. Genty, Instabilities, breathers and rogue waves in optics. Nat. Photonics 8, 755-764 (2014). doi:10.1038/nphoton.2014.220

12. E. Goulielmakis, M. Schultze, M. Hofstetter, V. S. Yakovlev, J. Gagnon, M. Uiberacker, A. L. Aquila, E. M. Gullikson, D. T. Attwood, R. Kienberger, F. Krausz, U. Kleineberg, Single-cycle nonlinear optics. Science 320, 1614-1617 (2008). doi:10.1126/science.1157846 Medline

13. A. Wirth, M. T. Hassan, I. Grguras, J. Gagnon, A. Moulet, T. T. Luu, S. Pabst, R. Santra, Z. A. Alahmed, A. M. Azzeer, V. S. Yakovlev, V. Pervak, F. Krausz, E. Goulielmakis, Synthesized light transients. Science 334, 195-200 (2011). doi:10.1126/science.1210268 $\underline{\text { Medline }}$

14. G. Krauss, S. Lohss, T. Hanke, A. Sell, S. Eggert, R. Huber, A. Leitenstorfer, Synthesis of a single cycle of light with compact erbium-doped fibre technology. Nat. Photonics 4, 3336 (2010). doi:10.1038/nphoton.2009.258

15. P. Krogen, H. Suchowski, H. Liang, N. Flemens, K.-H. Hong, F. X. Kärtner, J. Moses, Generation and multi-octave shaping of mid-infrared intense single-cycle pulses. Nat. Photonics 11, 222-226 (2017). doi:10.1038/nphoton.2017.34 
16. H. Liang, P. Krogen, Z. Wang, H. Park, T. Kroh, K. Zawilski, P. Schunemann, J. Moses, L. F. DiMauro, F. X. Kärtner, K.-H. Hong, High-energy mid-infrared sub-cycle pulse synthesis from a parametric amplifier. Nat. Commun. 8, 141 (2017). doi:10.1038/s41467017-00193-4 Medline

17. M. Tonouchi, Cutting-edge terahertz technology. Nat. Photonics 1, 97-105 (2007). doi:10.1038/nphoton.2007.3

18. Materials and methods are available as supplementary materials.

19. D. Strickland, G. Mourou, Compression of amplified chirped optical pulses. Opt. Commun. 55, 447-449 (1985). doi:10.1016/0030-4018(85)90151-8

20. A. Dubietis, G. Jonušauskas, A. Piskarskas, Powerful femtosecond pulse generation by chirped and stretched pulse parametric amplification in BBO crystal. Opt. Commun. 88, 437-440 (1992). doi:10.1016/0030-4018(92)90070-8

21. F. Smektala, C. Quemard, V. Couderc, A. Barthélémy, Non-linear optical properties of chalcogenide glasses measured by Z-scan. J. Non-Cryst. Solids 274, 232-237 (2000). doi:10.1016/S0022-3093(00)00215-5

22. H. Zhang, S. Virally, Q. Bao, L. K. Ping, S. Massar, N. Godbout, P. Kockaert, Z-scan measurement of the nonlinear refractive index of graphene. Opt. Lett. 37, 1856-1858 (2012). doi:10.1364/OL.37.001856 Medline

23. M. Z. Alam, I. De Leon, R. W. Boyd, Large optical nonlinearity of indium tin oxide in its epsilon-near-zero region. Science 352, 795-797 (2016). doi:10.1126/science.aae0330 $\underline{\text { Medline }}$

24. D. N. Nikogosyan, Nonlinear Optical Crystals: A Complete Survey (Springer Science \& Business Media, 2006).

25. S. Ghimire, A. D. DiChiara, E. Sistrunk, P. Agostini, L. F. DiMauro, D. A. Reis, Observation of high-order harmonic generation in a bulk crystal. Nat. Phys. 7, 138-141 (2011). doi:10.1038/nphys 1847

26. R. R. Gattass, E. Mazur, Femtosecond laser micromachining in transparent materials. Nat. Photonics 2, 219-225 (2008). doi:10.1038/nphoton.2008.47

27. A. Schiffrin, T. Paasch-Colberg, N. Karpowicz, V. Apalkov, D. Gerster, S. Mühlbrandt, M. Korbman, J. Reichert, M. Schultze, S. Holzner, J. V. Barth, R. Kienberger, R. Ernstorfer, V. S. Yakovlev, M. I. Stockman, F. Krausz, Optical-field-induced current in dielectrics. Nature 493, 70-74 (2013). doi:10.1038/nature11567 Medline

28. M. Krüger, M. Schenk, P. Hommelhoff, Attosecond control of electrons emitted from a nanoscale metal tip. Nature 475, 78-81 (2011). doi:10.1038/nature10196 Medline

29. U. Eichmann, A. Saenz, S. Eilzer, T. Nubbemeyer, W. Sandner, Observing Rydberg atoms to survive intense laser fields. Phys. Rev. Lett. 110, 203002 (2013).

doi:10.1103/PhysRevLett.110.203002 Medline

30. R. Adair, L. L. Chase, S. A. Payne, Nonlinear refractive index of optical crystals. Phys. Rev. B Condens. Matter 39, 3337-3350 (1989). doi:10.1103/PhysRevB.39.3337 Medline

31. www.refractiveindex.info. 
32. O. E. Martinez, Achromatic phase matching for second harmonic generation of femtosecond pulses. IEEE J. Quantum Electron. 25, 2464-2468 (1989). doi:10.1109/3.40630

33. N. Ishii, K. Kaneshima, K. Kitano, T. Kanai, S. Watanabe, J. Itatani, Sub-two-cycle, carrierenvelope phase-stable, intense optical pulses at $1.6 \mu \mathrm{m}$ from a $\mathrm{BiB}_{3} \mathrm{O}_{6}$ optical parametric chirped-pulse amplifier. Opt. Lett. 37, 4182-4184 (2012). doi:10.1364/OL.37.004182 Medline

34. Y. Deng, A. Schwarz, H. Fattahi, M. Ueffing, X. Gu, M. Ossiander, T. Metzger, V. Pervak, H. Ishizuki, T. Taira, T. Kobayashi, G. Marcus, F. Krausz, R. Kienberger, N. Karpowicz, Carrier-envelope-phase-stable, $1.2 \mathrm{~mJ}, 1.5$ cycle laser pulses at $2.1 \mu \mathrm{m}$. Opt. Lett. 37, 4973-4975 (2012). doi:10.1364/OL.37.004973 Medline 